\title{
INFLUÊNCIA DA DINÂMICA FAMILIAR NA QUALIDADE DE VIDA DE IDOSOS
}

\author{
Luciana Araújo dos Reis ${ }^{a}$ \\ Luana Araújo dos Reis ${ }^{b}$ \\ Gilson de Vasconcelos Torres ${ }^{c}$ \\ Kleyton Trindade Santos ${ }^{d}$
}

\begin{abstract}
Resumo
O presente estudo tem por objetivo investigar a influência da dinâmica familiar na qualidade de vida de idosos participantes de grupo de convivência. Trata-se de uma pesquisa de caráter analítica com delineamento transversal e amostra de 27 idosos independentes participantes de um Grupo de Convivência para a Terceira Idade no município de Jequié/BA. O instrumento de coleta constou de questões sócio-demográficas e condições de saúde, Apgar de Família e WHOQOL-OLD. Foi realizada análise estatística descritiva e aplicação da Correlação de Sperman, Teste de Mamn-Whitney e o Teste de Kruskal Wallis, uma vez que, os dados do presente estudo não apresentaram padrão de normalidade. O nível de significância adotado para os testes estatísticos foi de $5 \%$. O Coeficiente de correlação de Sperman mostrou uma correlação significativa entre as variáveis, comprometimento da dinâmica familiar e qualidade de vida apenas nas facetas, atividades presentes, passadas e futuras $(p \leq 0,001)$ e intimidade $(p \leq 0,003)$. De acordo com o teste de Mamn-Whitney e o teste de Kruskal-Wallis não houve diferença estatística entre dinâmica familiar e as variáveis, estado civil, escolaridade, ocupação, reside com, tipo de doença e medicação. Constatou-se no presente estudo que não houve diferença estatística entre dinâmica familiar e as variáveis, estado civil, escolaridade, ocupação, reside com, tipo de doença e medicação. Sendo encontrada uma correlação significativa entre as variáveis, comprometimento da dinâmica familiar e comprometimento da qualidade de vida apenas nas facetas, atividades presentes, passadas e futuras e intimidade.
\end{abstract}

Palavras-chave: Envelhecimento, família, qualidade de vida.

Correspondence Author: Luciana Araújo dos Reis - lucianauesb@yahoo.com.br.

a. Fisioterapeuta, Doutora em Ciências da Saúde/PPCSA-UFRN. Docente da Universidade Estadual do Sudoeste da Bahia e da Faculdade Independente do Nordeste.

b. Enfermeira, Mestrando em Enfermagem/UFBA.

c. Enfermeiro, Doutor em Enfermagem/EERP-USP, Docente do Programa de Pós-Graduação em Ciências da Saúde/CCSUFRN.

d. Fisioterapeuta. Mestrando em Enfermagem e Saúde/UESB. 


\title{
INFLUENCE OF FAMILY DYNAMICS IN THE QUALITY OF LIFE OF ELDERLY
}

\begin{abstract}
the present study aims to investigate the influence of family dynamics on the quality of elderly association group of participants. This is a survey of analytical character with cross-sectional design and a sample of 27 independent elderly participants of a Group Living for Seniors in Jequié/BA. The instrument consisted of socio-demographic and health status, Apgar Family and WHOQOL-OLD. Descriptive statistical analysis and application of the Spearman Correlation, Mamn-Whitney test and the Kruskal Wallis test was performed, since the data from this study were not statistically normal range. The Spearman correlation coefficient showed a significant correlation between the variables between family dynamics and impaired quality of life only in facets, activities present, past and future $(p \leq 0.001)$ and intimacy $(p \leq 0.003)$. According to Mamn-Whitney test and the Kruskal-Wallis test showed no statistical difference between family dynamics and variables, marital status, education, occupation, resides with, type of illness and medication. It was found in this study that there was no statistical difference between family dynamics and variables, marital status, education, occupation, resides with, type of illness and medication. Being found a significant correlation between the variables, impaired family dynamics and impaired quality of life only in facets, activities present, past and future and intimacy.
\end{abstract}

Keywords: Aging; family; quality of life.

\section{INTRODUÇÃO}

O Brasil apesar de ser um país jovem, vem demonstrando um perfil populacional do tipo de transição demográfica que sinaliza rápida mudança em termos de aumento vertiginoso do extrato idoso da população. Desde 1940, o grupo etário com 60 anos ou mais é aquele que, proporcionalmente mais tem crescido.(1)

Esse fenômeno de aumento no número de idosos na sociedade brasileira tem representado problemas sociais, econômicos e de saúde, uma vez que tanto os idosos quanto a sociedade não estão preparados para as mudanças decorrentes do envelhecimento. ${ }^{(2)} \mathrm{O}$ aumento tem feito das pessoas idosas ao viverem mais anos de vida possibilidade aumenta de sofrer o acometimento de uma doença crônico-degenerativa. As doenças crônico-degenerativas podem levar ao comprometimento da capacidade funcional tornando o idoso dependente. ${ }^{(3)}$
Com isto tem havido uma tendência a propiciar o isolamento social, na medida em que o idoso é visto como um ser improdutivo e dependente que se configura em gastos financeiros e representa necessidade de cuidados especiais. $\mathrm{O}$ isolamento social por sua vez, predispõe a inatividade física e, inúmeras são as repercussões na saúde desses indivíduos, limitando sobre tudo a capacidade funcional, comprometendo a sua qualidade de vida e impossibilitando que este indivíduo resida ou permaneça em sua residência sozinho, necessitando assim de cuidados, sobretudo de sua família.(4)

Com efeito, o papel da família, importante em qualquer estágio da vida, torna-se particularmente relevante durante períodos transitórios ou permanentes de menor capacidade física e/ou psíquica). (5) Essa funcionalidade familiar deve proporcionar ao idoso dependente uma condição satisfatória e 
participativa, visto que, o contrário poderá resultar em uma série de complicações derivadas de insuficiências materiais, psicológicas ou afetivas que acabam por interferir na qualidade de vida (QV) do idoso. ${ }^{(6)}$

Diante disso fica exposto à importância de se conhecer as condições de apoio familiar, em seu ambiente físico e psíquico, para um possível desenvolvimento de mecanismos de assistência domiciliária à saúde privilegiando o respeito à individualidade $\mathrm{e}$ privacidade do idoso, oferecendo-o um envelhecer favorável e acima de tudo melhorando sua qualidade de vida. Nesta perspectiva, este estudo tem por objetivo investigar a influência da dinâmica familiar na qualidade de vida de idosos participantes de grupo de convivência.

\section{MÉTODOS}

Trata-se de uma pesquisa de caráter analítica com delineamento transversal. O local de estudo foi um Grupo de Convivência para a Terceira Idade no município de Jequié/BA, sendo a amostra composta por 27 idosos.

O instrumento de coleta de dados foi constituído de questões sócio-demográficas e condições de saúde, pelo Apgar de Família(7) e pelo Questionário genérico WHOQOL-OLD. ${ }^{(8)}$ Os dados coletados foram organizados em banco de dados eletrônicos por meio de digitação em planilha do Programa Estatístico SPSS versão 20.0, sendo posteriormente realizada análise estatística descritiva e aplicação da Correlação de Sperman, Teste de Mamn-Whitney e o Teste de Kruskal Wallis, uma vez que, os dados do presente estudo não apresentaram padrão de normalidade. O nível de significância adotado para os testes estatísticos foi de $5 \%$.

Os procedimentos de coleta de informações foram instituídos após aprovação pelo Comitê de Ética da Universidade Estadual do Sudoeste da Bahia - UESB, obedecendo à Resoluçãor 96/96 (Protocolo $\left.n^{\circ} .005 / 2008\right)$ que trata das pesquisas realizadas em seres humanos. Para participar da pesquisa voluntariamente o idoso assinou um Termo de Consentimento Livre e Esclarecido.

\section{RESULTADOS}

Dos 27 idosos entrevistados, a média de idade foi de 70,78 anos $( \pm 4,35)$ anos. Houve uma predominância do sexo feminino $(85,19 \%)$, e maior distribuição do estado civil casado (37,04\%). Quanto ao nível de escolaridade $44,44 \%$ dos idosos eram analfabetos, sendo $81,48 \%$ aposentados com um salário mínimo.

Em relação à renda familiar $66,70 \%$ dos idosos possuíam até um salário mínimo, 29,60\% de 1 a 3 salários e 3,70\% de 4 a 6 salários. A presença de patologias foi relatada por $88,90 \%$ dos idosos, sendo a mais freqüente hipertensão $(25,90 \%)$, hipertensão e diabetes $(14,80 \%)$ e hipertensão e hipercolesterolemia (7,40\%). Quanto ao uso de medicação os mais citados foram os hipertensivos $(66,70 \%)$ e hipertensivos e insulina $(11,10 \%)$.

Tabela 1 - Distribuição dos idosos em relação às variáveis sócio-demográficas. Jequié/BA, 2014.

\begin{tabular}{lcc}
\hline \multicolumn{1}{c}{ VARIÁVEIS } & N & $\%$ \\
\hline Sexo & 23 & 85,19 \\
Feminino & 4 & 14,81 \\
Masculino & & \\
Idade & 11 & 40,73 \\
60 a 69 anos & 15 & 55,57 \\
70 a 79 anos & 1 & 3,70 \\
Acima de 80 anos
\end{tabular}


Tabela 1 - Distribuição dos idosos em relação às variáveis sócio-demográficas. Jequié/BA, 2014.

\begin{tabular}{lcc}
\hline \multicolumn{1}{c}{ VARIÁVEIS } & N & $\%$ \\
\hline Estado civil & 10 & 37,04 \\
Casado & 5 & 18,52 \\
Solteiro & 6 & 22,22 \\
Viúvo & 6 & 22,22 \\
$\quad$ Separado & & \\
Escolaridade & 8 & 29,63 \\
l grau completo & 1 & 3,70 \\
2 grau completo & 6 & 22,22 \\
Sabe ler e escrever & 12 & 44,44 \\
$\quad$ Analfabeto & & 81,48 \\
Ocupação & 22 & 11,11 \\
Aposentado & 3 & 3,70 \\
Pensão & 1 & 3,70 \\
$\quad$ Auxilio doença & 1 & $\mathbf{1 0 0 , 0 0}$ \\
Sem renda & $\mathbf{2 7}$ & \\
\hline Total & & \\
\hline
\end{tabular}

Verificou-se a respeito da dinâmica familiar que $85,19 \%$ dos idosos apresentaram uma boa funcionalidade familiar, $33,33 \%$ residiam com duas pessoas e $22,22 \%$ respectivamente moravam sozinhos ou com uma pessoa. Sendo que $22,22 \%$ residiam sozinhos e $14,81 \%$ com filhos e esposo (a) respectivamente, seguidos de $11,11 \%$ que dividiam o convívio domiciliar com o esposo (a) e neto ou apenas com os netos.

Tabela 2 - Distribuição dos idosos de acordo a dinâmica familiar, número de pessoas e com quem reside. Jequié/BA, 2014.

\begin{tabular}{lcc}
\hline & $N$ & $\%$ \\
\hline Dinâmica Familiar & & \\
O a 4 (elevada) & 1 & 3,70 \\
5 e 6 (moderada) & 3 & 11,11 \\
7 a 10 (boa) & 23 & 85,19 \\
Número de pessoas & & \\
Nenhuma & 6 & 22,22 \\
1 Pessoa & 6 & 22,22 \\
2 Pessoas & 9 & 33,33 \\
3 Pessoas & 4 & 14,81 \\
7 Pessoas & 2 & 7,41 \\
\hline
\end{tabular}


Tabela 2 - Distribuição dos idosos de acordo a dinâmica familiar, número de pessoas e com quem reside. Jequié/BA, 2014.

(conclusão)

\begin{tabular}{lcc}
\hline & N & \% \\
\hline Reside com & 4 & 14,81 \\
Filhos & 1 & 3,70 \\
Filho, neto e marido & 1 & 3,70 \\
Esposo e secretario & 3 & 11,11 \\
Esposo e neto & 1 & 3,70 \\
Esposo e entiado & 3 & 11,11 \\
Netos & 4 & 14,81 \\
Esposo(a) & 1 & 3,70 \\
Sobrinhos & 6 & 22,22 \\
Sozinha & 1 & 3,70 \\
Sobrinho, irmã, nora & 2 & 7,41 \\
Filho e neto & $\mathbf{2 7}$ & $\mathbf{1 0 0 , 0 0}$ \\
\hline Total & & (conclusáo) \\
\hline
\end{tabular}

Observou-se nos dados descritos que as médias e desvio-padrão para cada uma das facetas do
Whoqol-Old oscilam entre $61,93 \pm 24,52$ para as habilidades sensoriais e 78,00 $\pm 17,31$ para morte e morrer.

Tabela 3 - Distribuição das medidas de posição e dispersão das facetas do Whoqol-Old. Jequié/BA, 2014.

\begin{tabular}{lcccc}
\hline & \multicolumn{4}{c}{ MedidAS DE POSIÇão E DISPERSÃo } \\
\hline \multicolumn{1}{c}{ FACETAS DO WHOQOL-OLD } & N & MEDIA & $\begin{array}{c}\text { DESVIO } \\
\text { PADRÃo }\end{array}$ & IC (95\%) \\
Habilidades Sensoriais & 27 & 61,93 & 24,52 & 52,23 \\
Autonomia & 27 & 74,37 & 24,12 & 64,83 \\
Atividade presente, passada e futura & 27 & 70,63 & 12,35 & 65,74 \\
Participação Social & 27 & 74,11 & 11,84 & 69,43 \\
Morte e Morrer & 27 & 74,85 & 38,54 & 59,61 \\
Intimidade & 27 & 78,00 & 17,31 & 71,15 \\
\hline
\end{tabular}

De acordo com o teste de Mamn-Whitney e o teste de Kruskal-Wallis não houve diferença estatística entre dinâmica familiar e as variáveis, estado civil, escolaridade, ocupação, reside com, tipo de doença e medicação.
O Coeficiente de correlação de Sperman mostrou uma correlação significativa entre as variáveis, comprometimento da dinâmica familiar e comprometimento da qualidade de vida apenas nas facetas, atividades presentes, passadas e futuras $(p \leq 0,001)$ e intimidade $(p \leq 0,003)$. 
Tabela 4 - Distribuição dos Domínios do Whoqol-old em relação à Dinâmica familiar. Jequié/BA, 2014.

\begin{tabular}{lc}
\hline Habilidades Sensoriais & DINÂMICA FAMILIAR \\
S & 0,010 \\
P & 0,979 \\
Autonomia & \\
S & $-0,100$ \\
P & 0,606 \\
Atividade presente, passada e futura & \\
S & 0,610 \\
P & 0,001 \\
Participação Social & \\
S & 0,290 \\
P & 0,148 \\
Morte e morrer & \\
S & 0,070 \\
P & 0,727 \\
Intimidade & \\
S & 0,550 \\
P & 0,003 \\
\hline
\end{tabular}

\section{DISCUSSÃO}

De acordo com o presente estudo, houve diferença entre os gêneros, sendo mais predominante o sexo feminino. Isso confirma os resultados encontrados na literatura, pois como em outros paises do mundo, no Brasil há um número maior de mulheres na faixa etária idosa $(55,0 \%)$. Essa diferença se acentua com o aumento da idade: a razão de sexo é de 118 mulheres para cada 100 homens na faixa etária de 65-69 anos e de 141 para cada 100 no grupo de 80 anos ou mais. A expectativa de vida ao atingir 60 anos também acompanha o sexo, com mais 19,3 anos de vida, em média, para as mulheres contra 16,8 anos para os homens. ${ }^{(9)}$ A baixa escolaridade da maioria dos participantes no presente estudo, em que $44,44 \%$ dos idosos eram analfabetos, leva a necessidade de uma adequação das ações de educação em saúde, para que haja uma maior compreensão e motivação por parte dessas pessoas. Visto que algumas características como, analfabetismo, aposentadoria e alterações do estado nutricional podem levar a uma maior dependência do idoso em relação a outras pessoas. ${ }^{\left({ }^{10}\right)}$

No presente estudo $66,7 \%$ dos idosos relataram receber um salário mínimo. Isso enfatiza a necessidade da existência de programas voltados à prevenção e ao tratamento das doenças como subsidio para que o indivíduo possa se auto-cuidar, uma vez que a renda recebida por estes idosos, na maior parte dos casos, tem por finalidade cobrir todas as despesas mensais destes.

A hipertensão arterial, doença mais relatada pelo grupo de idosos do grupo de convivência, é considerada umas das causas mais comuns de morbidade e mortalidade prematura. ${ }^{(9)}$ Além da alta prevalência, constitui fator de risco para complicações cardiovasculares estando diretamente associada à 
incapacidade e dependência, repercutindo negativamente na qualidade de vida do idoso. ${ }^{(11)}$

A família é um sistema dinâmico em interação dialógica que pretende (ou deve pretender) ajudar a pessoa a desenvolver uma presença afetiva, responsável e livre no mundo.(5) Verificou-se a respeito da dinâmica familiar que $85,19 \%$ dos idosos apresentaram uma boa funcionalidade familiar. $\mathrm{O}$ reconhecimento da dinâmica familiar permite a detecção de disfunções e possibilita a intervenção precoce na busca do reequilíbrio dessa estrutura de relações e na melhoria da qualidade assistencial prestada ao idoso.

As famílias são, portanto, a origem de grande parte do suporte material, emocional e assistencial através de gerações. As constantes mudanças quanto à suplementação e demandas entre eles ajudam a estabelecer uma sólida reciprocidade confortável entre dar e receber. Também frequentemente pensa-se nos idosos unicamente como receptores. Isto nem sempre é verdadeiro, pois com o aumento do número de idosos e os avanços relacionados com a promoção da saúde no envelhecimento e com as intervenções assistenciais pode-se verificar que, atualmente com mais frequência, são eles responsáveis pelo provimento do suporte emocional e financeiro além do cuidado das crianças e da continuidade cultural e religiosa em muitas famílias. ${ }^{(12)}$ No presente estudo constatou-se que apenas $22,22 \%$ residiam sozinhos.

Os resultados deste estudo indicam que os idosos do Grupo de Convivência estudado no município de Jequié/BA apresentam bons escores de qualidade de vida para todos os domínios do Whoqol-Old quando comparados à pontuação máxima dos escores para cada domínio e às médias encontradas para os diferentes domínios da qualidade de vida na população estudada. Em relação à boa qualidade de vida percebida pelos idosos do estudo, vários fatores justificam tal resultado. A maioria dos idosos entrevistados realiza atividades físicas regulares e provavelmente se beneficiam com a mesma em vários aspectos. A atividade física está associada à melhora da saúde, à redução da morbidade e da mortalidade. (6) Ela aumenta a capacidade funcional e repercute positivamente num dos fatores mais preocupantes do envelhecimento: a dependência funcional. ${ }^{(13)}$

$\mathrm{O}$ conceito de $\mathrm{QV}$ está relacionado à auto-estima a ao bem-estar pessoal e abrange uma série de aspectos como a capacidade funcional, o nível socioeconômico, o estado emocional, a interação social, a atividade intelectual, o autocuidado, o suporte familiar, o próprio estado de saúde, os valores culturais, éticos e a religiosidade, o estilo de vida, a satisfação com o emprego e/ou com atividades diárias e o ambiente em que se vive. ${ }^{(14)}$

O Domínio atividades passadas, presentes e futuras, constituído por no qual os idosos relataram não estar satisfeitos com a possibilidade de continuar alcançando realizações pessoais e satisfação com o reconhecimento pela família e com o que alcançou em sua vida, foi afetado negativamente pelo comprometimento da dinâmica familiar. Desta forma, compreende-se ser fundamental para o planejamento assistencial adequado ao idoso, a compreensão de seu contexto familiar o que implica na compreensão das questões que envolvem a formação e a dinâmica de funcionamento das famílias em geral.

Em relação ao o Domínio Intimidade, em que são avaliadas questões relativas a companheirismo, capacidade de amar e se amado, foi encontrada diferença estatística com o comprometimento da dinâmica familiar. Dada à importância da família como órgão de apoio e saúde, a impossibilidade do idoso poder dispor destes recursos poderá levá-lo a situações de morbidade significativa, seja sob o prisma físico, psíquico ou social. Uma série de complicações derivadas de insuficiências materiais, psicológicas ou afetivas do grupo familiar em relação ao paciente idoso poderá levá-lo a situações de agressão potencial ou afetiva, física ou psíquica que acabam por interferir na qualidade de vida do idoso. ${ }^{(15)}$ 


\section{CONCLUSÕES}

Constatou-se no presente estudo que não houve diferença estatística entre dinâmica familiar e as variáveis, estado civil, escolaridade, ocupação, reside com, tipo de doença e medicação. Sendo encontrada uma correlação significativa entre as variáveis, comprometimento da dinâmica familiar e comprometimento da qualidade de vida apenas nas facetas, atividades presentes, passadas e futuras e intimidade.

\section{REFERÊNCIAS}

1. Veras R. Population aging today: demands, challenges and innovations. Rev. Saúde Pública 2009;43:548-54.

2. Giatti L, Barreto SM, Lima MFC. Condições de saúde, capacidade funcional, uso de serviços de saúde e gastos com medicamentos da população idosa brasileira: um estudo descritivo baseado na pesquisa Nacional por Amostra de domicílios. Cad. saúde pública. 2003;19(3);735743.

3. Del Duca GF, Thumé E, Hallal PC. Prevalência e fatores associados ao cuidado domiciliar de idosos. Rev Saude Publica 2011;45(1):113-20.

4. Gonçalves LHT, Alvarez AM, Sena ELS, Santana WS, Vicente FR. Perfil da familia cuidadora de idoso doente/fragilizado no contexto sociocultural de Florianopoles. Texto contexto enferm. 2006;15(4):570-577.

5. Pimenta GMF, Costa MADMC, Gonçalves LHT, Alvarez AM. Profile of the caregiver of dependent elderly family members in a home environment in the City of Porto, Portugal. Rev Esc Enferm USP. 2009;43(3)606-11.

6. Rodríguez-Sánchez E, Pérez-Peñaranda A, Losada-Baltar A, Pérez-Arechaederra, Gómez-Marcos MA, Patino-Alonso MC et al. Relationships between quality of life and family function in caregiver. BMC Fam Pract. 2011;12:1-7.
7. Silva MJ, Bessa MEP, Oliveira MC. Tamanho e estrutura familiar de idosos residentes em áreas periféricas de uma metrópoli. Cienc. enferm. 2004;10(1):31-39.

8. Pereira RJ, Cotta RMM, Franceschini SCC, Ribeiro RCL, Sampaio RF, Priore SE, Cecon PR. Contribuição dos domínios físico, social, psicológico e ambiental para a qualidade de vida global de idosos. Rev. bras. psicoter. 2006;28(1):27-38.

9. Reis LA, Mascarenhas CHM, Costa AN, Lessa RS. Estudo das condições de saúde de idosos em tratamento no setor de neurogeriatria da Clinica Escola de Fisioterapia da Universidade Estadual do Sudoeste da Bahia. Rev. baiana saúde pública. 2007;31(2):324-32.

10. Sampaio LS, Lessa RS, Reis LA, Torres GV. Prevalência de Patologias Ortopédicas em Idosos atendidos em Hospital Público no Município de Lajedo do Tabocal-BA. Rev. Enferm. Atual 2007 set/out;7(41):30-32.

11. Campolina AG, Dini PS, Ciconelli RM. Impacto da doença crônica na qualidade de vida de idosos da comunidade em São Paulo (SP,Brasil). Ciênc. saúde coletiva. 2011;16(6):2919-25.

12. Mendes MRSSB, Gusmão JL, Faro ACM, Leite RCBO. A situação social do idoso no Brasil:uma breve consideração. Acta paul. enferm. 2005;18(4):422-6.

13. Acree LS, Longfors J, Fjeldstad AS et al. Physical activity is related to quality of life in older adults. Health Qual Life Outcomes 2006; 4. Available at: http://www.hqlo.com/ content/4/1/37.

14. Alexandre TS, Cordeiro RC, Ramos LA. Factors associated to quality of life in active elderly. Rev. Saúde Pública. 2009;43(4):613-21.

15. Heinonen H, Aro AR, Aalto AM, Utela A. Is the evaluation of the global quality of life determined by emotional status? Qual Life Res. 2004;13(8):1347-56. 